\title{
Quantum Sensor for Nanoscale Defect Characterization
}

\author{
J. Kerski®, ${ }^{1,}{ }^{*}$ P. Lochner, ${ }^{1}$ A. Ludwig $\odot,{ }^{2}$ A.D. Wieck $\odot,{ }^{2}$ A. Kurzmann $\odot,{ }^{1,3}$ A. Lorke $\odot,{ }^{1}$ and \\ M. Geller@ ${ }^{1}$ \\ ${ }^{1}$ Faculty of Physics and CENIDE, University of Duisburg-Essen, Lotharstr. 1, Duisburg 47057, Germany \\ ${ }^{2}$ Chair for Applied Solid State Physics, Ruhr-Universität Bochum, Universitätsstr. 150, Bochum 44780, Germany \\ ${ }^{3}$ Solid State Physics Laboratory, ETH Zurich, Zurich 8093, Switzerland
}

(Received 22 July 2020; revised 16 November 2020; accepted 22 December 2020; published 12 February 2021)

\begin{abstract}
The optical and electronic properties of semiconductors are strongly affected by structural and stoichiometric defects. The precise incorporation of dopants and the control of impurities are essentially what makes semiconductors useful materials for a broad range of devices. The standard defect and impurity characterization methods are sensitive only on a macroscopic scale, like the most widely used method of deep-level transient spectroscopy (DLTS). We perform time-resolved measurements of the resonance fluorescence of a single self-assembled (In,Ga)As quantum dot (QD) at low temperatures (4.2 K). By pulsing the applied gate voltage, we are able to selectively occupy and unoccupy individual defects in the vicinity of the dot. We address the exciton transition of the QD with a tunable diode laser. Our timeresolved measurements exhibit a shift of the resonance energy of the optical transition. We attribute this to a change of the electric field in the dot's vicinity, caused by electrons tunneling from a reservoir to the defect sites. Furthermore, we are able to characterize the defects concerning their position and activation energy by modeling our experimental data. Our results thus demonstrate how a quantum dot can be used as a quantum sensor to characterize the position and activation energy of individual shallow defects on the nanoscale.
\end{abstract}

DOI: 10.1103/PhysRevApplied.15.024029

\section{INTRODUCTION}

The optical and electronic properties of semiconductors are strongly affected by structural and stoichiometric defects [1]. In particular, the incorporation of impurities as dopants alters the intrinsic properties of semiconductors and enables their use in (opto)electronic devices [2]. On the other hand, for emerging semiconductor-based quantum-information technologies, defects can also be detrimental. They constitute nonradiative recombination centers and lead to dephasing in nearby quantum emitters $[3,4]$. Therefore, a precise characterization of defect states and their influence on quantum emitters, with $\mathrm{nm}$ spatial, $\mu \mathrm{eV}$ energy and single-electron-charge resolution, is highly desirable. Here, we use a solid-state quantum emitter under resonant optical excitation as a sensitive electrometer [5] to probe defect states in the surrounding, nominally undoped semiconductor material. The experiments

\footnotetext{
*jens.kerski@uni-due.de

Published by the American Physical Society under the terms of the Creative Commons Attribution 4.0 International license. Further distribution of this work must maintain attribution to the author(s) and the published article's title, journal citation, and DOI.
}

reveal not only the position of the defect states along the growth direction with nanometer resolution but also their activation energies. This is achieved by measurements of the defects' occupation dynamics, even for very shallow states that are indiscernible in conventional defect spectroscopy [6,7]. The results highlight the significance of impurities for the optical properties of single-photon emitters. They also provide us with a deep insight into the diffusion and segregation processes of dopants [8] and thus pave the way towards solid-state emitters of indistinguishable single photons with lifetime-limited spectral purity [9-14].

Quantum-information technologies [15,16], such as quantum computing [17,18] and cryptography [19,20], are the most famous and challenging visions of quantum mechanics in future real-world applications. However, besides the control and manipulation of quantum states, another relevant application is the use of quantummechanical systems for metrology and quantum sensing $[21,22]$. A wide range of different quantum sensors exists [21], where the sensor is used to measure a physical quantity with high precision. Single spins in the negatively charged nitrogen vacancy center in diamond can be used for ultralow magnetic field detection [22-24], high-precision temperature measurements [25], or position tracking in living cells [26]. Sensing electric fields 
has been realized in single electron transistors [27] by transport through a nanometer constriction $[28,29]$ or by optical means, using, for instance, exciton transitions as a quantum-mechanical two-level system in a solid-state quantum emitter [3,4,30,31].

We use here the excitonic transition of a single (In,Ga)As self-assembled quantum dot in resonant excitation as a highly sensitive quantum sensor (i.e., a nanoscale electrometer) for time-resolved measurements of the charge carrier tunneling from the $n+$ back contact to the nearby defect states. A boxcarlike evaluation method of the electron tunneling transients allows us to obtain precisely the position and the activation energy of the shallow defects $(<100 \mathrm{meV})$. Common techniques to investigate defects in semiconductors, e.g., junction spectroscopy [32], optical capacitance [33], or deep-level transient spectroscopy (DLTS) [6,7] typically make use of space charges near a Schottky or $p-n$ junction. The junction (Schottky or $p-n$ ) forms in these techniques a depletion region and measures a large number of defects with activation energies typically greater than $100 \mathrm{meV}$; due to the measurement principle, where the thermally activated emission and capture process defines the time constant of charging and discharging transients [34,35].

In our quantum-sensing approach, we make use of the electric field dependence of the excitonic transitions in a quantum dot. The permanent dipole moment of the exciton [36] leads to a linear quantum-confined Stark effect. Therefore, minute shifts in the exciton transition energy will be proportional to changes in the local electric field. This way, we are able to detect the electric field of a single charge, trapped in a defect state near the quantum dot.

Within the framework of the determined activation energies and positions in the band structure, we are not able to conclusively answer the question about the nature of the defects. However, we would like to suggest that they might be donorlike defects. In this case, injection with electrons would lead to neutralization of the initially positively charged defects. Nevertheless, our methods and model would also apply to other defects that can change their charging state in response to an external electric field.

For the measurement technique used, the quantum dot is embedded in a $p-i-n$ diode structure with a highly $n$-doped layer as electrical back contact and a Schottky contact as top gate (see Fig. 1 and Appendix A). We use a confocal microscope setup in a bath cryostat at a temperature $T=$ 4.2 K (see Kurzmann et al. [37] and Appendix B for more details). The quantum dot is excited resonantly by a tunable diode laser and the emitted photons are detected by a photo diode and lock-in technique in differential reflection (DR) and on an avalanche photodiode (APD) in resonance fluorescence (RF).

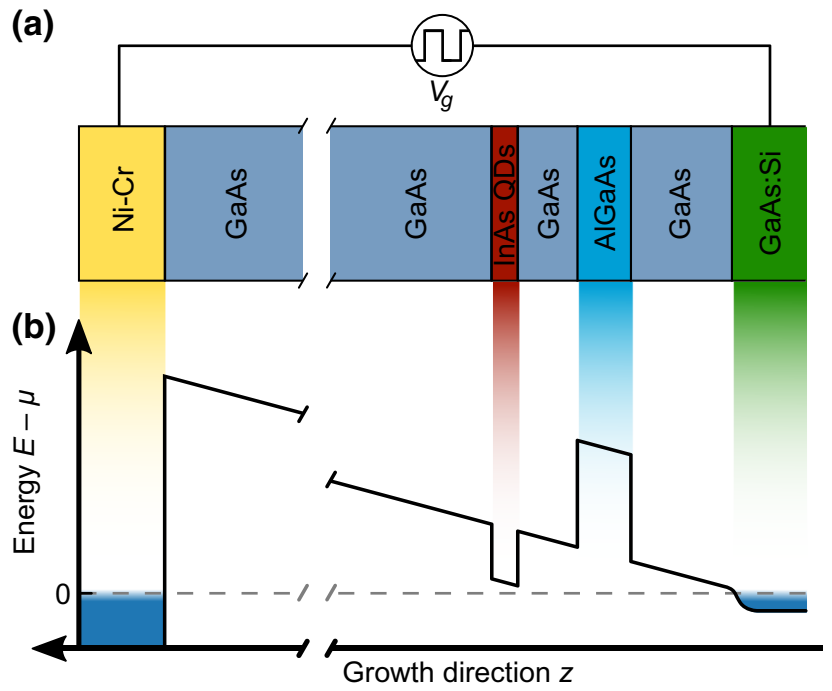

FIG. 1. Simplified illustration of sample structure and its conduction-band edge. (a) InAs QDs are embedded in a Schottky diode, formed by a metallic Ni-Cr gate and a highly silicondoped GaAs back contact. Application of a voltage $V_{g}$ between gate and back contact allows the QDs to be selectively occupied and their optical transitions to be tuned. (b) Corresponding conduction-band edge. A layer of $(\mathrm{Al}, \mathrm{Ga}) \mathrm{As}$ acts as a tunneling barrier between the QDs and the back contact.

\section{RESULTS AND DISCUSSION}

Figure 2(a) shows the exciton transition energy of a single self-assembled quantum dot (QD1) versus gate voltage $V_{g}$ in differential reflection. The energy is given in terms of a relative shift, where $0 \mu \mathrm{eV}$ corresponds to a laser energy of $1.302962 \mathrm{eV}$. As DR is based on a lockin technique that shifts the optical transition in and out of resonance with a square-pulsed gate voltage, the resonant condition is seen twice, shifted by the modulation amplitude of $100 \mathrm{mV}$. In this figure, however, we show only one feature of the measurement data. The given voltage value on the $x$ axis corresponds to the upper value of the square-pulsed voltage. This allows a direct comparison with the data recorded with resonant fluorescence. For the complete data set and further explanations on the method see Appendix B.

The resonance energies monotonically shift upwards with increasing gate voltage. However, this quantumconfined Stark shift exhibits three distinct discontinuities (labeled $A, B$, and $C$ ) that occur at specific gate voltages (at around $100 \mathrm{mV},-60 \mathrm{mV}$, and $-150 \mathrm{mV}$, respectively), which correspond to the occupation voltages of single defects in the vicinity of the $\operatorname{dot}[3,31]$. A fourth defect $D$ at an even smaller occupation voltage $\left(V_{g} \approx-300 \mathrm{mV}\right)$ is not visible in the DR scan, but can be identified in the nonequilibrium measurements below, as they allow characterization of the defects with much higher sensitivity. Features of a second quantum dot are visible at gate 

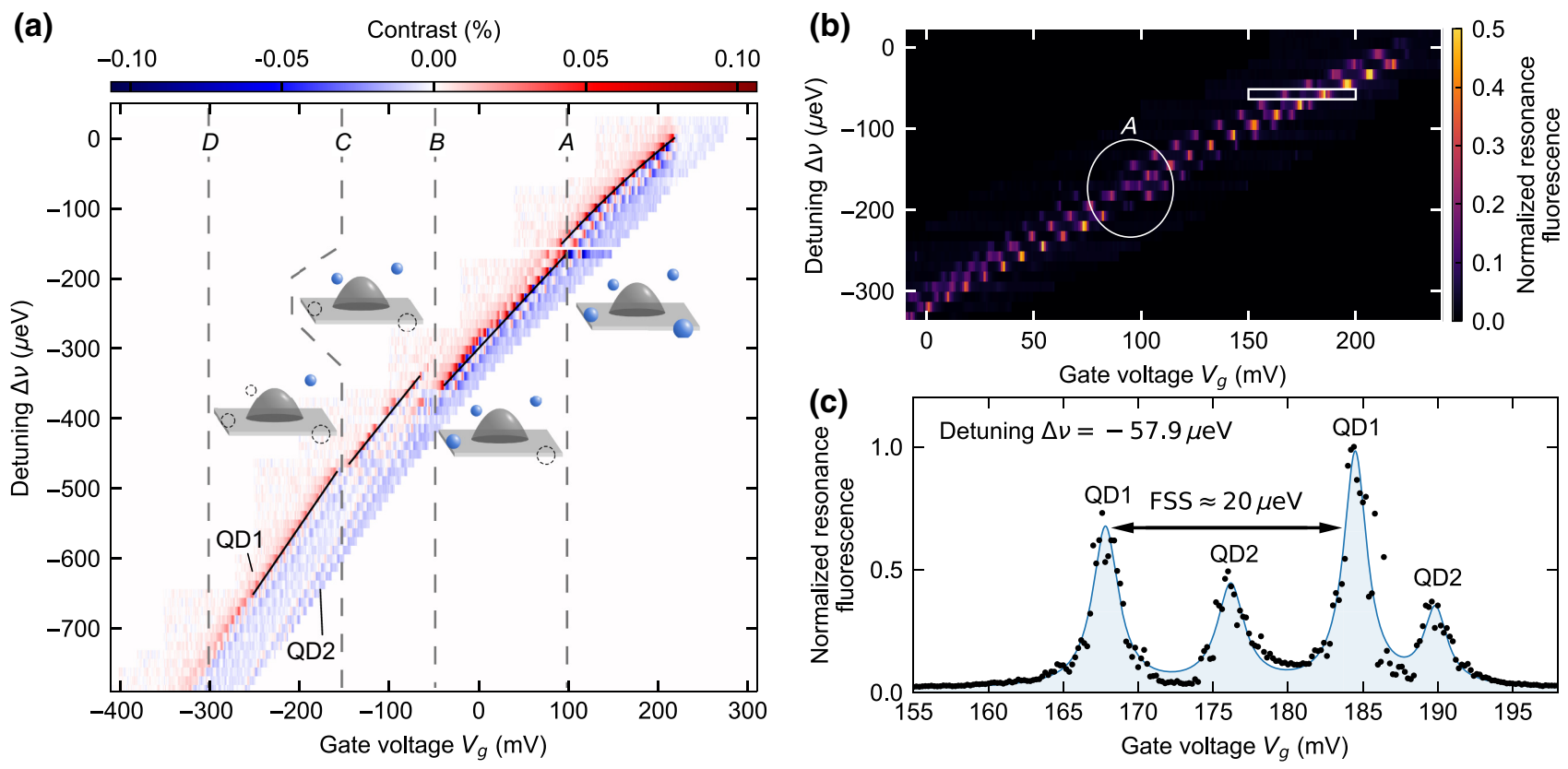

FIG. 2. Resonant exciton spectra of single quantum dots. The excitation laser energy is given in $\mu \mathrm{eV}$ detuning, where zero detuning corresponds to $1.302962 \mathrm{eV}$. (a) Differential reflection characteristics of two quantum dots (QD1 and QD2). QD1 is used as the nanoscale electrometer. The continuous shift of the resonance energy with applied gate voltage (i.e., the quantum-confined Stark effect) is interrupted by three distinct gaps $A, B, C$, indicating single occupation events in the vicinity of the QD. (b) High-resolution $\mathrm{RF}$ of the exciton transitions. The discontinuity $A$ can be observed at $100 \mathrm{mV}$. (c) Normalized resonance-fluorescence spectra of QD1 and QD2 at $-57.9 \mu \mathrm{eV}$ detuning [white box in (b)] with Lorentzian fits (blue lines).

voltages below $V_{g}=-50 \mathrm{mV}$ in Fig. 2(a), marked as QD2. Above $V_{g}=100 \mathrm{mV}$, the energy difference between QD1 and QD2 decreases with increasing gate voltage due to the difference in the Stark shift of both dots.

The DR technique allows it to obtain an overview over a large parameter range [Fig. 2(a)], but it is limited in energy and time resolution. Therefore, we use the more time consuming technique of resonance fluorescence (RF, see Appendix B) in the following. As an example, Fig. 2(b) shows an RF scan in the vicinity of occupation event $A$. The abrupt shift in resonance energy is again visible [white circle in Fig. 2(b)]. Furthermore, the spectral features of both dots QD1 and QD2 can clearly be observed in the RF spectra, as shown, for example, in Fig. 2(c). For the chosen detuning of $-57.9 \mu \mathrm{eV}$ [see white box in Fig. 2(b)], the fine-structure-split exciton transitions of QD1 are excited at gate voltages of 168 and $184 \mathrm{mV}$, the ones of QD2 at 176 and $190 \mathrm{mV}$. The intensity of QD2 is approximately $50 \%$ lower compared to QD1, as QD1 is in the center of the optical focus.

The occupation of the different defects manifests itself very differently in a shift of the optical transition of the QD. For defect $B$, for example, it is very large, for defect $D$ it cannot be resolved by DR, and for defect $A$ it even occurs in the opposite direction. This considers the fact that the energy shift due to the change of the electric field caused by the occupation of the defect is not trivial.
This is because the field vector has both a vertical and a horizontal component at the location of the quantum dot. The influence of an electric field in vertical direction is experimentally easily accessible via the observed shift due to the gate voltage. The only unknown factor is on which side of the quantum dot the defect is located and thus whether the defect amplifies or compensates the electric field that falls across the diode. However, the influence of the horizontal component cannot be determined in our experiment. Rather, for a precise explanation of the observed energy shifts one would have to consider that the electric fields of the different defects compensate each other as soon as they are occupied. For example, it would be possible that the horizontal component of the electric field change of defect $A$ is opposite to that of defect $B$. The observed energy increase would then result from the vertical component of the field of defect $A$. Due to the above-mentioned points, we do not consider the energy shift itself as a suitable quantity for determining the positions of the defects and the precise explanation of the energy shifts is beyond the scope of this paper.

In a next step, the brighter QD1 is used as a quantum electrometer to determine the activation energies and positions of the defect states. We assume that the defects are donors and can be in two charging states, i.e., they are either positively charged (unoccupied) or electrically neutral (occupied with a single negative charge). The 
occupation and unoccupation takes place by electron tunneling between the highly $n$-doped back contact and the defects. These assumptions are in agreement with previous studies [3] and are further supported by our own data evaluation below.

The measurement technique is shown in Fig. 3. Starting from a preparation voltage $V_{\text {prep }}=-680 \mathrm{mV}$, at $t=0$ a detection pulse bias $V_{\text {det }}$ of variable amplitude is applied [Fig. 3(a)]. In an $n$-shot measurement, the time-resolved resonance-fluorescence signal is recorded for each $V_{\text {det }}$, see Fig. 3(b). For sufficient long times $(t>400 \mu \mathrm{s})$, the resulting spectrum, shown in Fig. 3(c), corresponds to the equilibrium spectrum shown in Fig. 2(c). At shorter times $(t \approx 100 \mu \mathrm{s})$, however, the spectra exhibit additional resonances that are absent in the equilibrium state, for example at $V_{\text {det }}=152 \mathrm{mV}$ and $V_{\text {det }}=206 \mathrm{mV}$ [red and green line in Fig. 3(b)]. As the amplitude of the voltage pulses, $V_{\text {det }}-V_{\text {prep }}$, spans the entire range of occupation voltages of the defects $A-D$, the time evolution of the RF signal reflects some of the possible occupation sequences, depicted in Fig. 3(d). We emphasize that for all detection voltages $V_{\text {det }}$ the same state (all defects are occupied) is reached for long periods of time. The variation serves only for the spectral investigation of the quantum dot.

For a more detailed evaluation, we choose some characteristic time traces, highlighted by colored lines in Fig. 3(b). Other time traces are not considered, as they are either associated with QD2 or are simple replicas of the QD1 resonances, shifted by the fine-structure splitting of $20 \mu \mathrm{eV}$. The RF time evolution at $V_{\text {det }}=152 \mathrm{mV}$ [red line, bottom trace in Fig. 3(e)] is characterized by an instantaneous onset and a continuous decay to zero that corresponds to a single exponential function. We therefore attribute this signal to the initial state, where all defects are unoccupied [see insets in Fig. 3(e)]. The signal at a detection voltage of $206 \mathrm{mV}$ [green line, middle trace in Fig. 3(e)] increases more slowly, goes through a maximum at $t \approx 100 \mu \mathrm{s}$ and then decreases again to almost zero. This indicates an intermediate state where some but not all defects are occupied. The time trace that leads to the equilibrium configuration ( $184 \mathrm{mV}$, blue line) is shown in the top panel of Fig. 3(e). Remarkably, there is a considerable latency before the RF signal turns on. This behavior cannot be described by simple parallel or sequential occupation events of the different defects, which would result in a polynomial increase of the signal at $t \approx 0$, similar to the one at $V_{\text {det }}=206 \mathrm{mV}$, middle panel. The $100 \mu$ s dark time before the onset of the equilibrium signal therefore suggests a more complicated arrangement and rearrangement of charges before the final configuration can be reached.

So far, all possible occupation sequences are probed simultaneously by the application of a large gate-voltage pulse, spanning all four defect states. In order to investigate the dynamics of a specific single defect, a different pulse (a)

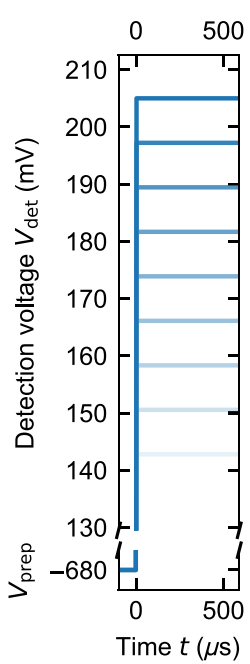

(b)

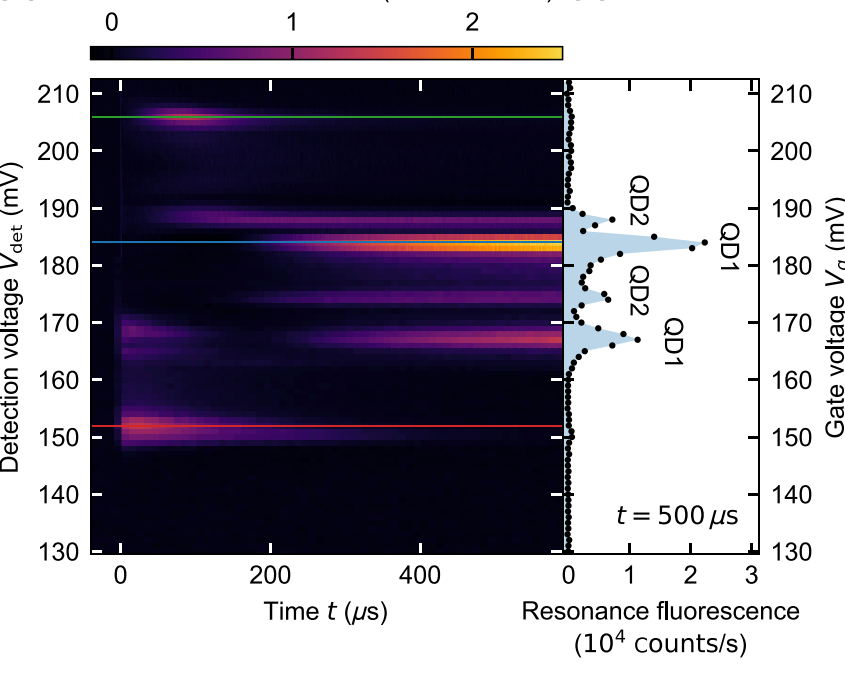

(d)
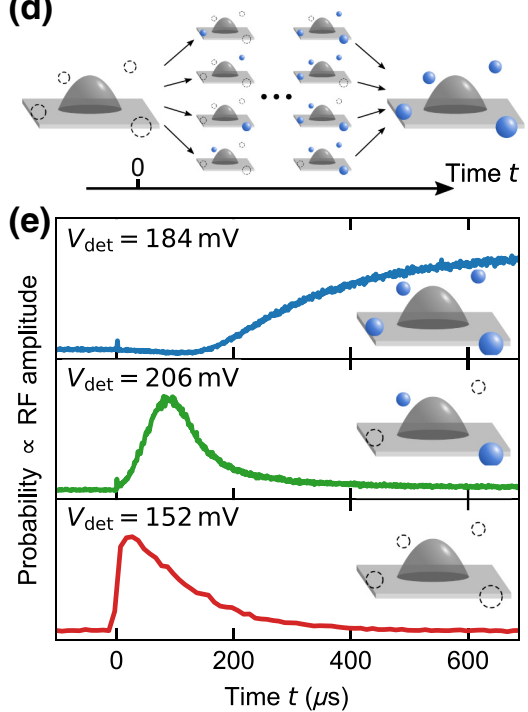

FIG. 3. Time-resolved detection of different defect charge configurations. (a) Illustration of the measurement by pulsing the gate voltage. The preparation voltage $V_{\text {prep }}$ for $t<0$ is fixed at $V_{\text {prep }}=-680 \mathrm{mV}$, while the detection voltage $V_{\text {det }}$ for $t \geq 0$ is increased stepwise to scan the exciton transitions of QD1 and QD2. (b) Time-resolved resonance-fluorescence amplitude as a function of $V_{\text {det }}$. Transient signals are clearly observed. The fluorescence amplitude at $t=500 \mu \mathrm{s}$, shown in (c), corresponds to the equilibrium case [cf. Fig. 2(c)]. (d) Illustration of possible occupation sequences of the defects in the vicinity of the QD. The system is prepared in an initial state, where all defects are unoccupied for $t \leq 0$ and relaxes by intermediate states (partly occupied defects) into the thermodynamic equilibrium (completely occupied defects). (e) Three transients as line cuts taken from (b), displaying the probability evolution of different charge-carrier configurations inside the defect states (blue line, completely occupied defects; green line, partly occupied defects; red line, completely unoccupied defects). 

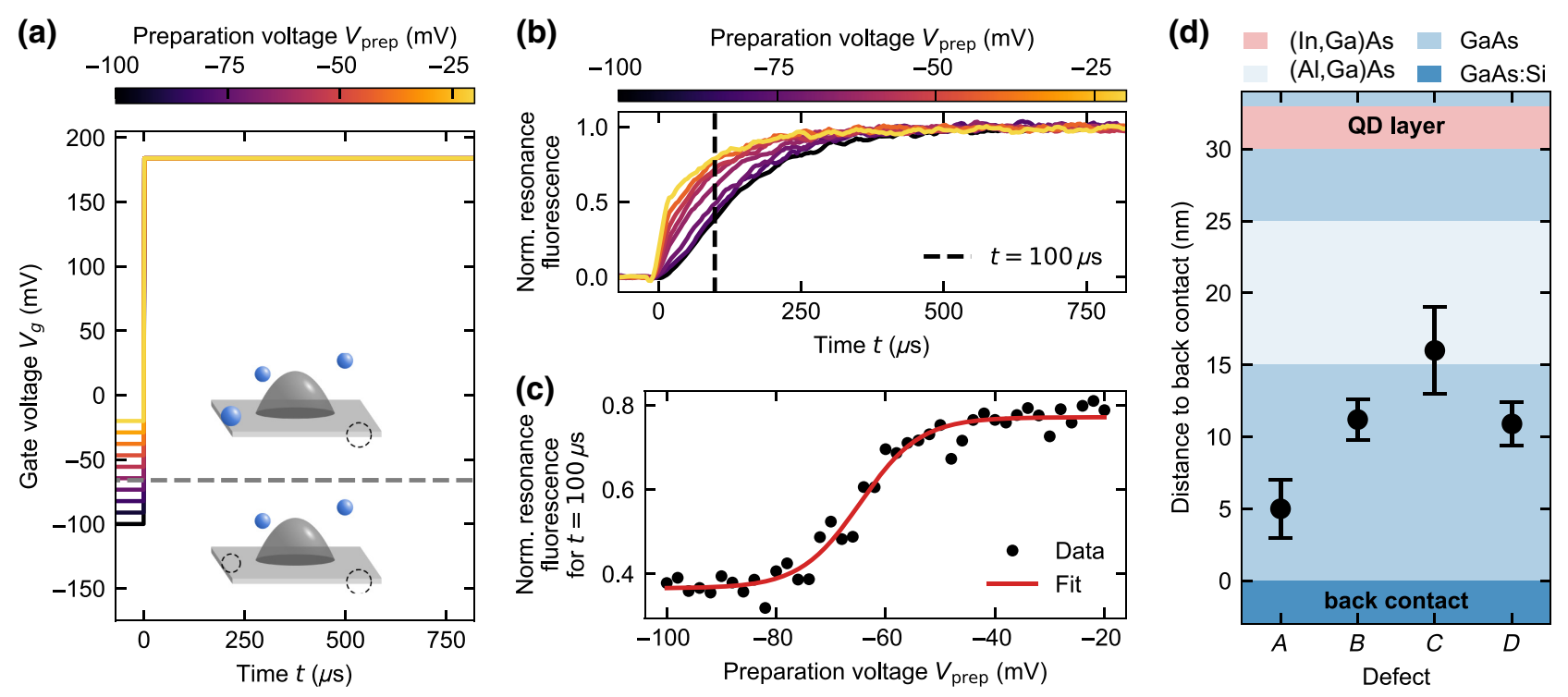

FIG. 4. Defect characterization by time-resolved resonance-fluorescence spectroscopy. (a) Illustration of the measurement sequence. The preparation voltage $V_{\text {prep }}$ for $t<0$ is increased stepwise, while the detection voltage $V_{\text {det }}$ for $t \geq 0$ is fixed at $184 \mathrm{mV}$. (b) Timeresolved RF measurement for different preparation voltages between $V_{\text {prep }}=-100 \mathrm{mV}$ and $-20 \mathrm{mV}$, including the occupation voltage of defect $B$ at $V_{g} \approx-65 \mathrm{mV}$. (c) Normalized resonance-fluorescence signal at time $t=100 \mu \mathrm{s}$ from the transients in (b). The solid red line displays a fit to the data using Eq. (1). (d) Schematic representation of the sample structure, including the $n$-doped GaAs back contact, the undoped GaAs and (Al,Ga)As barriers, and the (In,Ga)As layer, where the quantum dots are located. Data points show the positions of the four different defects $A-D$. The defect positions are determined by the lever arm $\lambda$, which is a fitting parameter in Eq. (1).

technique is used, as schematically shown in Fig. 4(a). Here, defect $B$ is probed, which has an occupation voltage of $V_{T}=-65 \mathrm{mV}$ [see Fig. 2(a) and Appendix B]. Accordingly, the preparation voltage is varied across $V_{T}=-65$ $\mathrm{mV}$ [dashed line in Fig. 4(a)]. The detection voltage is kept constant at $V_{\text {det }}=184 \mathrm{mV}$ (all defects occupied) and the $\mathrm{RF}$ signal of the electrometer QD1 is monitored as before.

Figure 4(b) shows characteristic transients for some selected preparation voltages between $V_{\text {prep }}=-100$ and $-20 \mathrm{mV}$. The data shows a clear transition from a situation where both defects $(A$ and $B$ ) need to be occupied ( $V_{\text {prep }}<-65 \mathrm{mV}$, black and purple lines) to one where only defect $A$ has to be filled ( $V_{\text {prep }}>-65 \mathrm{mV}$, orange and yellow lines). It can also be seen that this transition is not abrupt. For a more quantitative evaluation, the normalized $\mathrm{RF}$ signal at a representative time $t=100 \mu \mathrm{s}$ is plotted in Fig. 4(c). As in the boxcar evaluation of DLTS data [7], this resonance-fluorescence signal $I\left(V_{\text {prep }}, t=100 \mu \mathrm{s}\right)$ reflects the occupation probability $n_{B}\left(V_{\text {prep }}\right)$ of defect $B$ at the chosen time $t=100 \mu \mathrm{s}$ (see Appendix C). The occupation energy of a single defect is expected to be sharp. The reason that the occupation probability of defect $B$ changes smoothly lies in the thermal distribution of the back contact. As shown by the red solid line in Fig. 4(c), the data can be well fitted by a Fermi function.

This makes it possible to determine two characteristic parameters of this single defect: its activation energy $E_{B}$, i.e., its energetic position below the GaAs conduction-band edge, and its location - more specifically its distance $d_{B}$ from the back contact.

To determine $d_{B}$, we make use of the fact that the conversion factor between gate voltage $V_{g}$ and energy, $\Delta E=$ $e \Delta V_{g} / \lambda$, is given by the lever arm $\lambda=d_{\text {gate }} / d_{B}$, where $d_{\text {gate }}=170 \mathrm{~nm}$ is the distance between the back contact and the top gate [38]. Recently, we had shown that this allows one to use a quantum-dot state as an energy probe to map out the Fermi distribution in the back contact [37]. Here, we take the inverse approach and use a Fermi-Dirac fit for the resonance-fluorescence intensity

$$
I\left(V_{\text {prep }}, t=100 \mu \mathrm{s}\right) \propto \frac{1}{\exp \left[\frac{e\left(V_{T}-V_{\text {prep }}\right) / \lambda}{k_{B} T}\right]+1}
$$

to determine (via $\lambda$ ) the separation between the defect and the back contact. The resulting fit for defect $\mathrm{B}$ is shown as a solid line in Fig. 4(c), with $V_{T}=-65 \mathrm{mV}$ being the voltage at which the chemical potential of the back contact is in resonance with the defect state. We find $d_{B}=11.2 \pm$ $1.4 \mathrm{~nm}$ for defect $B$. From similar evaluations of defects $A$, $C$, and $D$, we obtain $d_{A}=5 \pm 2 \mathrm{~nm}, d_{C}=16 \pm 3 \mathrm{~nm}$, and $d_{D}=10.9 \pm 1.5 \mathrm{~nm}$ (see Appendix D). These values are summarized in Fig. 4(d), which depicts the location of the defects with respect to the back contact, the quantum-dot layer, and the $(\mathrm{Al}) \mathrm{GaAs}$ layers in between. 
With the such determined spatial positions of the defects, we can now also derive their energetic positions below the GaAs conduction-band edge, by comparing $V_{T}$ to the gate voltage $V_{S}$, where flat band condition is achieved $E=e\left(V_{S}-V_{T}\right) / \lambda$ (see also Fig. 9 of Appendix C). The flat band voltage is simply given by the surface Schottky barrier height $e V_{S}=600 \mathrm{meV}$ [37]. From an evaluation of all four defects (see Appendix D), we find $E_{A}=15 \pm 7 \mathrm{meV}, E_{B}=44 \pm 6 \mathrm{meV}, E_{C}=69 \pm$ $16 \mathrm{meV}, E_{D}=58 \pm 8 \mathrm{meV}$.

Note that all defects that lead to discontinuities in the dot spectra are located between the QD layer and the back contact. More precisely, three of them are located in the GaAs layer above the back contact and the fourth defect, $C$, is located at the interface between $\mathrm{GaAs}$ and $(\mathrm{Al}, \mathrm{Ga}) \mathrm{As}$ layer. Because of the high purity of the epitaxially grown material, we identify the highly Si-doped back contact as the most likely source of defects. Si-dopant segregation in GaAs is commonly observed [40] and in general limits the performance of epitaxially grown devices until today [41]. However, only the activation energy of defect $A$ is in reasonable agreement with the silicon donor level, $6 \mathrm{meV}$ below the GaAs conduction-band edge [42]. So far, we have not been able to identify the nature of the other defects. However, little is known about other impurities that may exhibit more than one charging state. Note that the defect states $B$ and $D$ are located at the same distances from the back contact and as the activation energy of defect $B$ is $14 \mathrm{meV}$ smaller, therefore it is possible that these states correspond to the same defect, energetically separated by a Coulomb charging energy. And even less is known about the corresponding energy levels. Possible candidates for such impurities would be vacancies, interstitials, and combinations thereof (maybe even combined with substitutional impurities as the famous $\mathrm{N}-V$ centers in diamond). Also, it should be noted that - because InAs has a 7\% larger lattice constant than GaAs - the quantum dots are surrounded by a field of substantial crystal strain that might shift the energy levels of any impurity considerably. We believe that our results raised some interesting questions regarding the origin of the spectroscopic fingerprints of the impurities, which warrants further investigations. Without additional experimental and theoretical work, however, we do not want to further speculate on the origin of these chargeable electronic states.

In conclusion, we use a solid-state emitter, a selfassembled QD, as a quantum sensor to characterize shallow defects on the nanoscale. Time-resolved resonancefluorescence measurements enable us to associate discontinuities in the Stark shift of the exciton transitions to single defects in the vicinity of the quantum dot. These defects are occupied by electron tunneling from the back contact of the Schottky diode structure. Evaluation of the time evolution of one exciton transition gives access to their positions along the growth direction and their activation energies.
This method can be used more generally for nanoscale defect characterization in different materials with different quantum emitters $(\mathrm{N}-V$ centers in diamond, defects in $h$ $\mathrm{BN}$, etc.). Moreover, this nanoscale defect characterization gives further information about dopant segregation, occurring in crystal-growth techniques [like, for instance, MBE and metal organic chemical vapor deposition (MOCVD)], hence, it could be an additional tool for adjusting the growth process to suppress the appearance of defects in semiconductors and insulators.

\section{ACKNOWLEDGMENTS}

This work is funded by the Deutsche Forschungsgemeinschaft (DFG, German Research Foundation) - ProjectID 278162697-SFB 1242 and the individual Research Grants No. GE2141/5-1 and No. LU2051/1-1. Ar.L. and A.D.W. acknowledge gratefully support of DFG-TRR160, BMBF-Q.Link.X 16KIS0867, and the DFH/UFA CDFA-05-06.

J.K., P.L., M.G., A.K., and A.L. planned the experiments. Ar.L. designed the heterostructure. Ar.L. and A.D.W. grew and A.K. fabricated the sample. J.K. and P.L. performed the measurements. J.K. evaluated the data. A.L. supervised the data analysis and M.G. supervised the experimental work. J.K., A.L., and M.G. wrote the manuscript.

\section{APPENDIX A: SAMPLE AND DEVICE FABRICATION}

The investigated sample material is grown by molecular beam epitaxy and represents a Schottky diode with embedded InAs QDs. Figure 5(a) displays schematically the MBE-grown layer sequence and Fig. 5(b) shows the calculated conduction-band edge and Fermi energy of our sample for zero applied gate voltage along the growth direction.

In detail, on top of a GaAs substrate a superlattice consisting of AlAs and GaAs is deposited, followed by a $300 \mathrm{~nm}$ layer of $\mathrm{GaAs}$ to provide a smooth surface for the low-defect growth of the diode structure. The back contact is formed by depositing a $50 \mathrm{~nm}$ thick layer of highly Si-doped GaAs. The quantum dots are separated from the back contact by a tunneling barrier, which consists of $15 \mathrm{~nm} \mathrm{GaAs}, 10 \mathrm{~nm}$ of $\mathrm{Al}_{0.34} \mathrm{Ga}_{0.66} \mathrm{As}$, and another $5 \mathrm{~nm}$ of GaAs. The quantum dots are formed by depositing 1.9 monolayers of InAs while interrupting the rotation of the sample holder. The dots are then capped with $2.5 \mathrm{~nm}$ of GaAs, followed by a $600^{\circ} \mathrm{C}$ flush to shift their emission wavelength to about $950 \mathrm{~nm}(1.3 \mathrm{eV})$. An additional layer of $27.5 \mathrm{~nm} \mathrm{GaAs}$, a second 100-nm-thick superlattice of AlAs and GaAs, and a layer of $10 \mathrm{~nm} \mathrm{GaAs}$ suppress undesirable currents through the diode.

The sample surface is partly covered with a transparent Schottky gate of $7 \mathrm{~nm} \mathrm{Ni-Cr}$. Au-Ge, and $\mathrm{Ni}$ are 


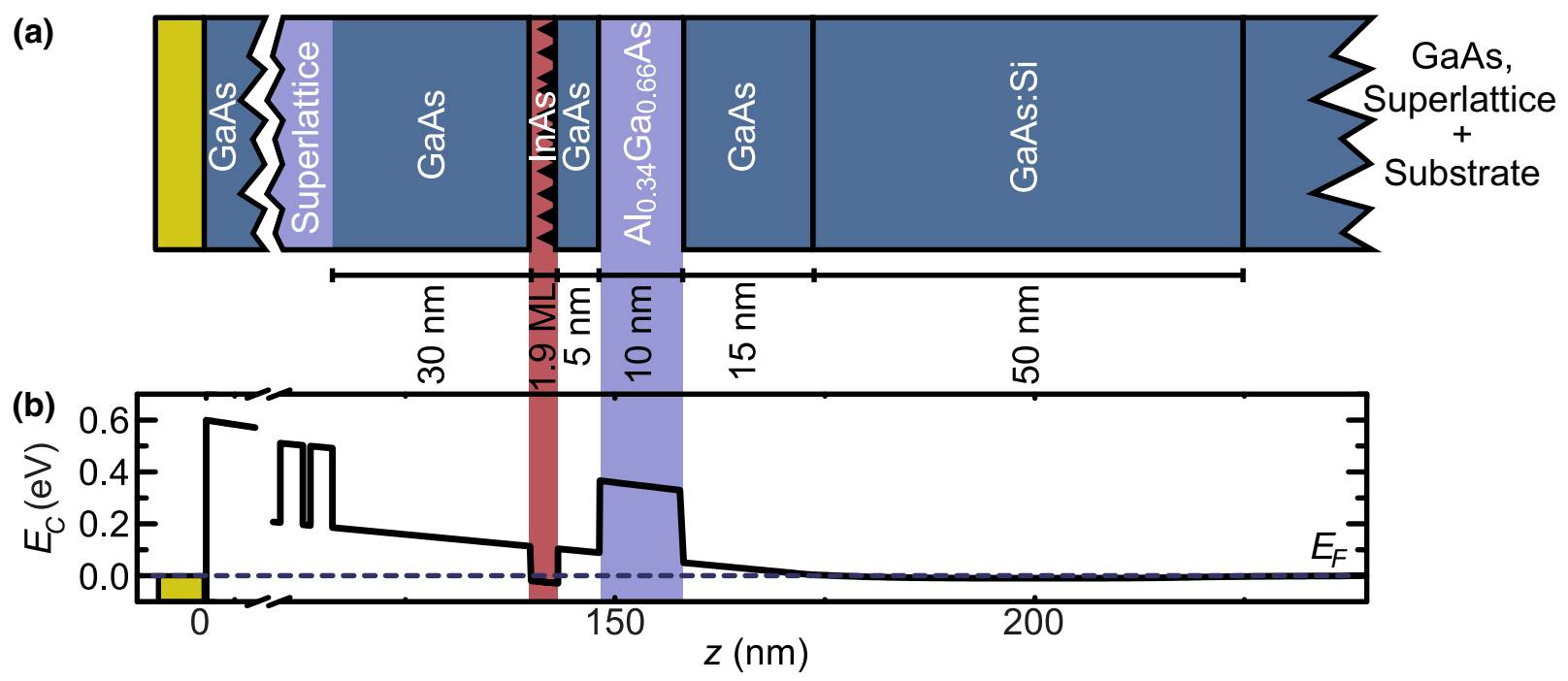

FIG. 5. Sample structure. (a) Illustration of the layer structure of the investigated sample. The quantum-dot layer (red) and the tunneling barrier (purple) are highlighted. The Ni-Cr gate (yellow) and the highly Si-doped GaAs back contact form a Schottky diode. (b) Conduction-band edge and Fermi energy along growth direction for zero applied gate voltage, calculated with the Poisson solver [39].

deposited and annealed using photolithography to contact the Si-doped GaAs, which acts as the Ohmic back contact. The conduction-band edge energy at the gate interface $(z=0)$ of $600 \mathrm{meV}$ relative to the Fermi energy $E_{F}$ of the Ohmic back contact $(175 \mathrm{~nm}<z<225 \mathrm{~nm})$ is the built-in Schottky barrier.

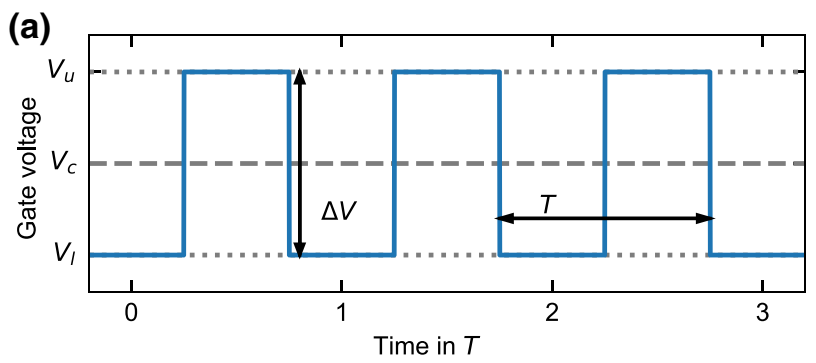

\section{APPENDIX B: OPTICAL MEASUREMENTS}

To perform optical measurements, the sample is mounted in a bath cryostat cooled with liquid helium to $4.2 \mathrm{~K}$. The resonant exciton excitation is performed with a frequency-stabilized tunable diode laser and a confocal microscope setup. Using an objective lens with

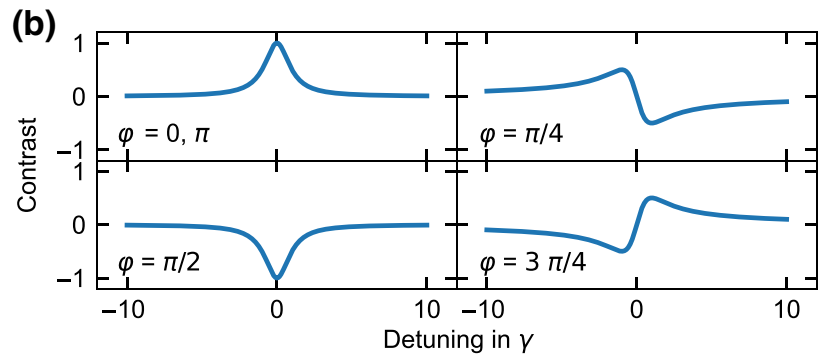

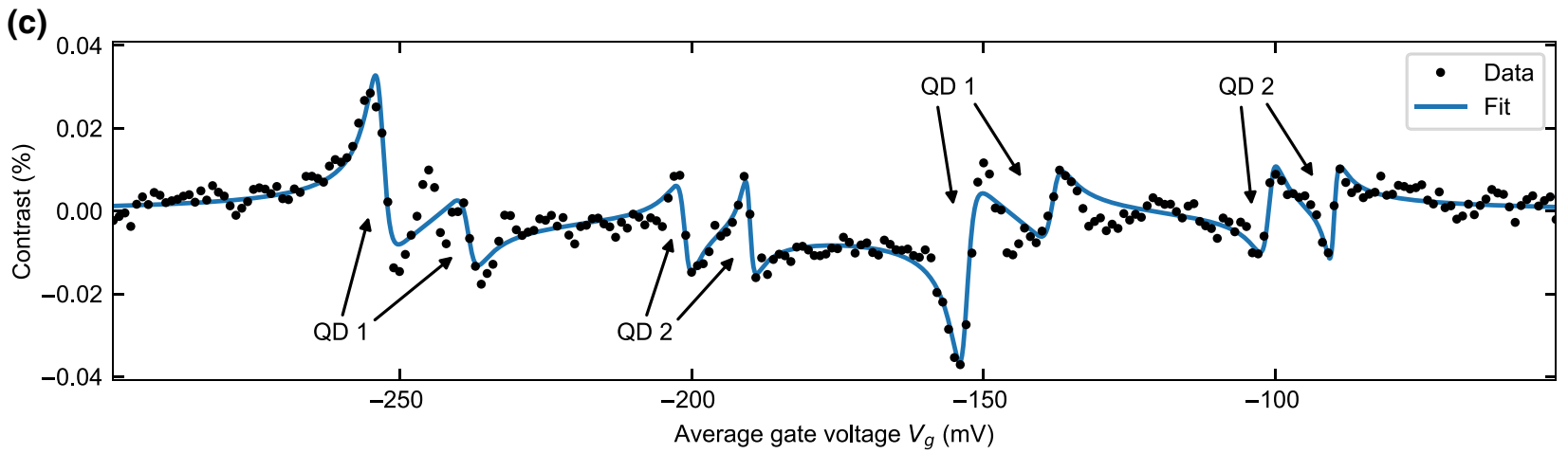

FIG. 6. Differential reflection. (a) Square-pulsed voltage with amplitude $\Delta V$ brings optical transition periodically in and out of resonance. (b) Depending on phase difference $\varphi$ between QD and reflected laser photons, different line shapes can occur [43]. (c) Exemplary DR spectrum for an irradiated laser energy detuning of approximately equal to $-562 \mu \mathrm{eV}$. Two optical transitions each of QD1 and QD2 are visible. Due to the DR method every optical transition is observed twice with opposite sign and separated by the amplitude $\Delta V=100 \mathrm{mV}$. The blue line is a model calculation based on adjusting the amplitude and phase for both QDs [see (b)]. 
$\mathrm{NA}=0.65$, single quantum-dot resolution is achieved by a focal spot size of about $1 \mu \mathrm{m}$. We present data taken with differential reflection [cf. Fig. 2(a)] and resonance fluorescence [cf. Fig. 2(b)].

\section{Differential reflection}

Differential reflection is an optical characterization method in which electron-hole pairs are resonantly excited in the quantum dot, and the photons emitted during recombination are detected. However, the irradiated photons can also be reflected both inside and on the surface of the sample without interacting with the QD. To separate these two types of photons, DR uses a lock-in procedure. In this method, a square-pulsed gate voltage is used to periodically shift the optical transition into and out of resonance with the irradiated laser [see Fig. 6(a)]. The two values of the gate voltage are separated by the amplitude $\Delta V$, which is $100 \mathrm{mV}$ in our experiment.

Figure 6(c) shows an example of a spectrum acquired with DR. The energy of the laser is fixed and the offset of the square-pulsed gate voltage is gradually increased. This way, each optical transition gets in resonance twice, once with the upper gate voltage value and once with the lower one. Although the line of the optical transition is actually Lorentz shaped, it can deviate greatly in DR [see Fig. 6(b)]. This is caused by interference with the reflected photons that are also detected and depends on the phase relationship $\varphi$ between the signals [43].

The interpretation of such a spectrum is very complicated and often only possible by additional spectra with different laser energies. For this reason we decide to give only a simplified representation of our measurement data in the main text by showing only the feature that is observed by resonance at the upper voltage value. An overview of the complete data set is given in Fig. 7.

\section{Resonance fluorescence}

For resonance-fluorescence measurements, the crosspolarization technique is used, where one polarizer polarizes the laser light linearly before it is guided to the quantum-dot sample. A second polarizer absorbs the reflected laser light, giving a laser background suppression of up to $10^{7}$. The photons emitted by the quantum dot exhibit a different polarization and are partially transmitted. The signal is detected with an avalanche photodiode. Time-resolved resonance-fluorescence measurements (cf. Figs. 3 and 4) are recorded by analyzing the signal of the APD with a time-to-digital converter with a time resolution of 81 ps. Furthermore, the gate voltage is pulsed according to the specific experiment. For a high signalto-noise ratio, the measurement is repeated in an $n$-shot measurement.

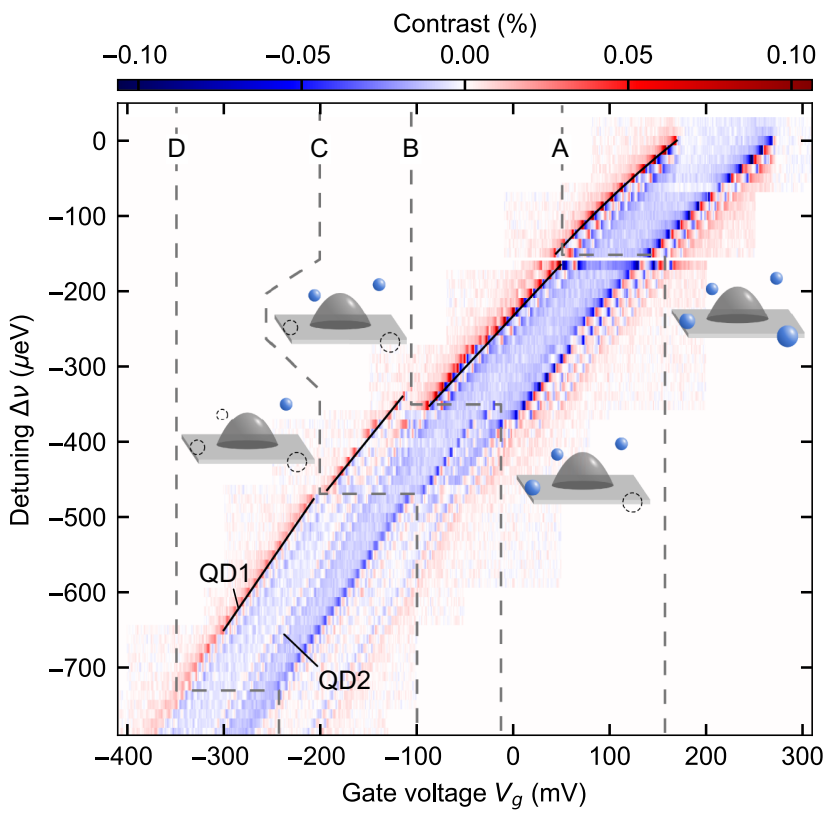

FIG. 7. Differential reflection characteristics of QD1 and QD2. QD1 is used as the nanoscale electrometer. The continuous shift of the resonance energy with applied gate voltage (i.e., the quantum-confined Stark effect) is interrupted by three distinct gaps $A, B, C$, indicating single occupation events in the vicinity of the QD. The DR signal is recorded with a voltage modulation of $100 \mathrm{mV}$. Therefore, all spectral features are seen twice, with inverted contrast (see top scale).

\section{APPENDIX C: MODEL OF THE RELAXATION PROCESS}

The activation energies and positions of the defects are obtained from a Fermi-Dirac fit [Eq. (1) in the main text] to the resonance-fluorescence amplitude, taken from the transients $I\left(V_{\text {prep }}, t\right)$ in Fig. 4(b) in the main text. We present here a more detailed explanation of the underlying model.

Let us first consider the occupation of a single defect in time. For a given time $t$, there is a probability for the defect $\alpha$ to be either occupied $P_{\alpha}(t)$ or unoccupied $P_{\neg \alpha}(t)$. This can be described by the expression

$$
P_{\alpha}(t)+P_{\neg \alpha}(t)=1 .
$$

We assume that the defect becomes occupied by an electron, which originates from the back contact and tunnels through a barrier. This process is taken into account by the tunneling rate $\gamma_{\alpha}=1 / \tau_{\alpha}$. If the occupation process begins at $t=0$, the probability for the defect to be unoccupied is given by

$$
P_{\neg \alpha}(t)=P_{0} \exp \left(-t / \tau_{\alpha}\right)
$$

where $P_{0}$ is a constant, corresponding to the probability of the defect to be unoccupied before the tunneling occurs. $P_{\alpha}$ can be derived from Eq. (C1). This expression is valid 
for low enough temperatures and detection voltages higher than the defect's tunneling voltage $V_{\text {det }}>V_{T}$.

Within the experiment in Fig. 4(b), more than one defect is occupied. So let us consider now two defects ( $\alpha$ and $\beta$ ), which are occupied simultaneously. These two defects are assumed not to interact with each other, e.g. have independent tunneling rates $1 / \tau_{\alpha}$ and $1 / \tau_{\beta}$. In this approach, the state of the system is described by the probabilities $P_{\alpha \wedge \beta}, P_{\alpha \wedge \neg \beta}, P_{\neg \alpha \wedge \beta}$ and $P_{\neg \alpha \wedge \neg \beta}$, which correspond to the situation, that both, only one or none of the defects are occupied. The probabilities for the state of the system can be derived from the probabilities for the single defects (e.g., $P_{\alpha \wedge \beta}=P_{\alpha} \times P_{\beta}$ ). The equilibrium resonance-fluorescence transients $I\left(V_{\text {prep }}, t\right)$, which are investigated in Fig. 4(b), are proportional to the probability $P_{\alpha \wedge \beta}$, where both defects are occupied for $t \rightarrow$ $\infty$.

Finally, we have to consider that one defect at higher gate voltages (here, defect $\beta$ ) is always unoccupied at the beginning of the transient $(t=0)$ and completely occupied in equilibrium $(t \rightarrow \infty)$. The other defect (here, defect $\alpha$ ) is completely occupied in equilibrium, however, the occupation state at the beginning of the transient depends on the preparation voltage $V_{\text {prep }}$. This is taken into account by the fact that the constant in Eq. (C2) equals 1 for defect $\beta$ and depends on the preparation voltage $P_{0}=P_{0}\left(V_{\text {prep }}\right)$ for defect $\alpha$ :

$$
\begin{aligned}
P_{\alpha \wedge \beta}\left(V_{\text {prep }}, t\right)= & P_{\alpha}\left(V_{\text {prep }}, t\right) \times P_{\beta}(t) \\
= & P_{0}\left(V_{\text {prep }}\right)\left[\exp (-t / \tau)-\exp \left(-t / \tau_{\alpha}\right)\right] \\
& +1-\exp \left(-t / \tau_{\beta}\right) \\
= & P_{0}\left(V_{\text {prep }}\right) \times c_{1}(t)+c_{2}(t),
\end{aligned}
$$

with $1 / \tau=1 / \tau_{\alpha}+1 / \tau_{\beta}$ and two time-dependent constants $c_{1}(t)$ and $c_{2}(t)$. So the probability $P_{\alpha \wedge \beta}$ is for a given time $t$ proportional to the constant $P_{0}\left(V_{\text {prep }}\right)$. Since this constant corresponds to the probability that the defect $\alpha$ is unoccupied before the pulse occurs at $t=0$, it can also be expressed by the probability of occupation $n\left(V_{\text {prep }}\right)$ :

$$
n\left(V_{\text {prep }}\right)=1-P_{0}\left(V_{\text {prep }}\right) .
$$

This occupation probability at the beginning of the transient is different for every defect (set by the preparation voltage) and depends on two characteristic parameters, i.e., its position in the diode structure and the activation energy $E_{a}$. As the defect $\alpha$ is in thermal equilibrium with the back contact at $t=0$, a bound state in the defect with the energy $E$ is occupied according to the Fermi-Dirac distribution of the back contact $n(E)$. The energy of the state $E\left(V_{\text {prep }}\right)$ in respect to the chemical potential of the back contact consists of two contributions. The first one reflects the stationary electric fields and is therefore called electrostatic energy $E_{\mathrm{el}}$. It takes the built-in Schottky voltage $V_{S}$ and

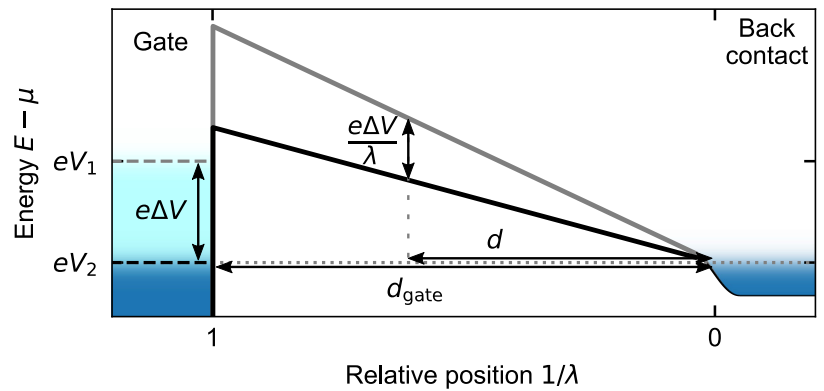

FIG. 8. Illustration of the lever arm. The lever arm $\lambda$ is defined for a specific position in the diode structure as the ratio of the distance between the back contact and the specific position $d$ and the distance between the back contact and the gate $d_{\text {gate }}$.

the preparation voltage $V_{\text {prep }}$ into account. Since the voltages are applied over the whole sample structure (between back contact and gate), there is also a geometric factor $\lambda$, which incorporates the vertical position of the defect (see Fig. 8). It is called lever arm $\lambda=d_{\text {gate }} / d_{\text {state }}$ [38]. The constants $d_{\text {gate }}$ and $d_{\text {state }}$ correspond to the distance between the back contact and the gate or the defect of the state, respectively. The second contribution is the activation energy $E_{a}$ of defect $\alpha$, i.e., the difference of the state's energy and the conduction-band edge:

$$
\begin{aligned}
E\left(V_{\text {prep }}\right) & =E_{e l}-E_{a} \\
& =e \frac{V_{S}-V_{\text {prep }}}{\lambda}-E_{a} \\
& =\frac{e}{\lambda}\left(V_{T}-V_{\text {prep }}\right),
\end{aligned}
$$

with the elementary charge $e$ and the tunneling voltage $V_{T}=V_{S}-\lambda E_{a} / e$.

Using Eq. (C5) and the Fermi-Dirac distribution function, one finds an expression for the voltage-dependent probability of occupation of the defect state $\alpha$ :

$$
\begin{aligned}
n\left(V_{\text {prep }}\right) & =\frac{1}{\exp \left[\frac{E\left(V_{\text {prep }}\right)}{k_{B} T}\right]+1}, \\
& =\frac{1}{\exp \left[\frac{e}{\lambda k_{B} T}\left(V_{T}-V_{\text {prep }}\right)\right]+1},
\end{aligned}
$$

with the thermal energy $k_{B} T$. The influence of activation energy and lever arm are illustrated in Fig. 9. The left panels show the conduction-band edge, including two defect states for two different preparation voltages. The right panels show the occupation probability of the states for a continuous scan of voltage.

The occupation probability of two defects with the same activation energy but different lever arms differs in a scan of the preparation voltage, illustrated in Fig. 9(a). The defect having a larger lever arm [Fig. 9(a), green level] 

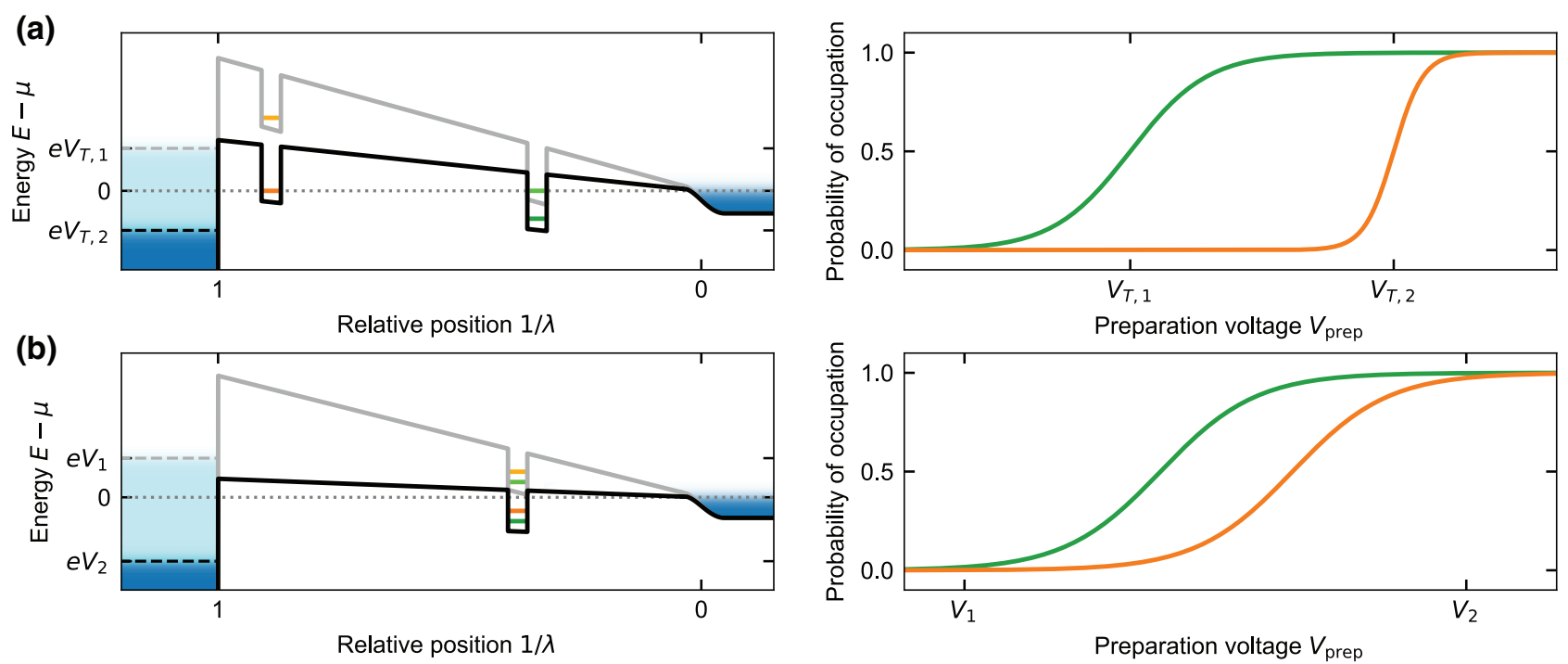

FIG. 9. Illustration of the influence of lever arm and activation energy on the occupation probability. Left panels show conductionband edge for two different preparation voltages. Right panels show the occupation probability $n\left(V_{\text {prep }}\right)$ as a function of the preparation voltage. (a) Increasing the lever arm $\lambda$ moves the relative position of the defect closer to the back contact $(1 / \lambda<0)$. The orange defect level has a small lever arm (left panel) and exhibits larger voltage range (right panel), where the defect level is in resonance with the chemical potential of the back contact. The lever arm also influences the charging voltage $V_{T, 1}$ and $V_{T, 2}$. (b) A defect at the same position with different activation energy (left panel) shifts the charging positions of the defect level without changing the resonant voltage range (right panel).

is closer to the back contact and therefore gets in resonance with its chemical potential for a smaller preparation voltage $V_{T, 1}$. A larger lever arm results also in a smaller energy change for a given change in voltage. Therefore, the voltage range, where the defect is occupied, is larger in comparison to defects (the Fermi distribution is "smeared out"), which have a larger distance from the back contact [orange line in Fig. 9(a) with occupation voltage $V_{T, 2}$ ].

Figure 9(b) demonstrates the influence of the activation energy on the occupation voltages. A smaller activation energy (orange line) shifts the occupation voltage to a larger preparation voltage; however, the occupation range stays unchanged, visible for the orange and green line of the occupation distribution.

From Eqs. (C3), (C4), and (C6) follows

$$
\begin{aligned}
P_{\alpha \wedge \beta}\left(t, V_{\text {prep }}\right) & =n_{\alpha}\left(V_{\text {prep }}\right) c_{1}(t)+c_{2}(t) \\
& =\frac{c_{1}(t)}{\exp \left[\frac{e}{\lambda k_{B} T}\left(V_{T}-V_{\text {prep }}\right)\right]+1}+c_{2}(t) .
\end{aligned}
$$

In conclusion, one finds a simple expression for the data in Fig. 4(b) for a given time $t$, which can be fitted to it and allows determination of the position of the occupied defect in growth direction as well as its activation energy. This relation is independent of the choice of $t$, just changing the constants $c_{1}(t)$ and $c_{2}(t)$. The considerations are more general as they also apply for situations of more than two defects. An approach using a set of master equations is also possible and will give the same expression.

\section{APPENDIX D: FURTHER DATA OF DEFECT CHARACTERIZATION}

We investigate four defects in this study. In the main text the data and its evaluation is shown exemplary for defect $B$. In the following, we present the data for the defects $A$, $C$, and $D$ [Figs. 10(a)-10(c), respectively].

The measurements differ to the one in the main text by different values for the preparation voltage $V_{\text {prep }}$. In the case of defect $A$, the measurement suffered from a low signal-to-noise ratio. Therefore, we evaluate and present here a different data set for defect $A$, where the detection voltage is $169 \mathrm{mV}$. Using this voltage, we probe the resonance fluorescence of the second fine-structure split line of the exciton transition [cf. Fig. 3(c)].

In the left panels of Fig. 10 transients for selected preparation voltages are shown. Dashed lines indicate the time, where the evaluation of the amplitudes takes place. In the right panels the corresponding amplitudes are shown for all transients, as well as a fit using Eq. (C7). Defect $D$ especially causes a big difference in the normalized resonance-fluorescence amplitude [see Fig. 10(c)]. This allows us to characterize a defect, whose impact on the energy scale is smaller than our resolution in equilibrium measurements of the differential reflection amplitude [cf. Fig. 2(a)]. 

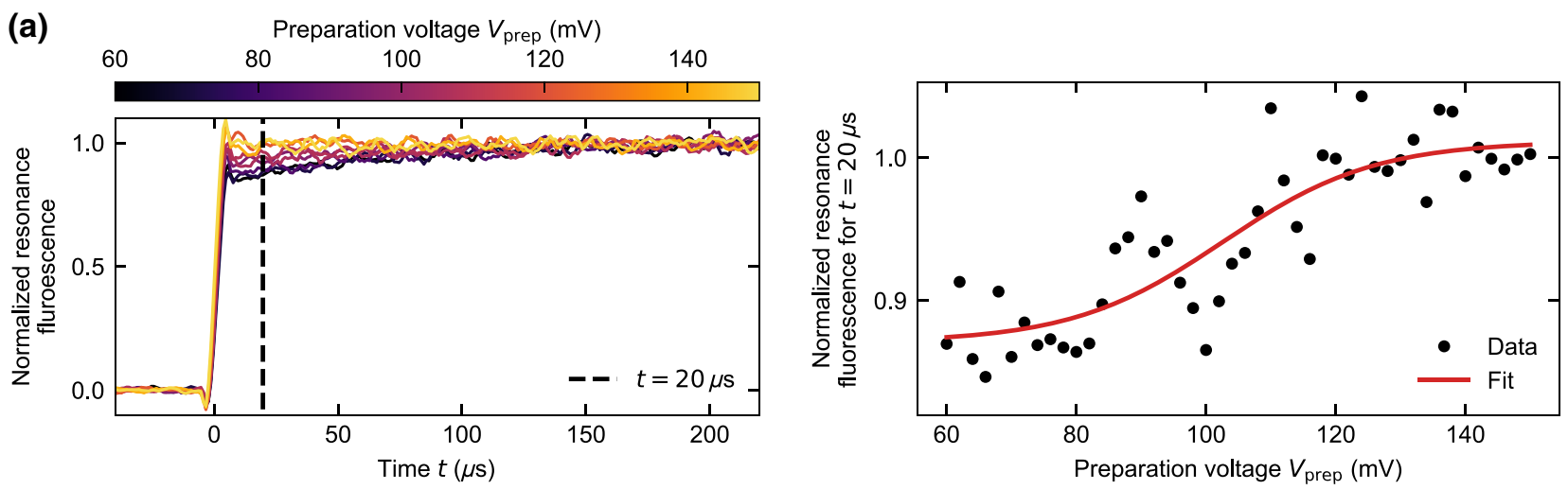

$\begin{array}{lcr}\text { (b) } & \text { Preparation voltage } V_{\text {prep }}(\mathrm{mV}) & -100\end{array}$
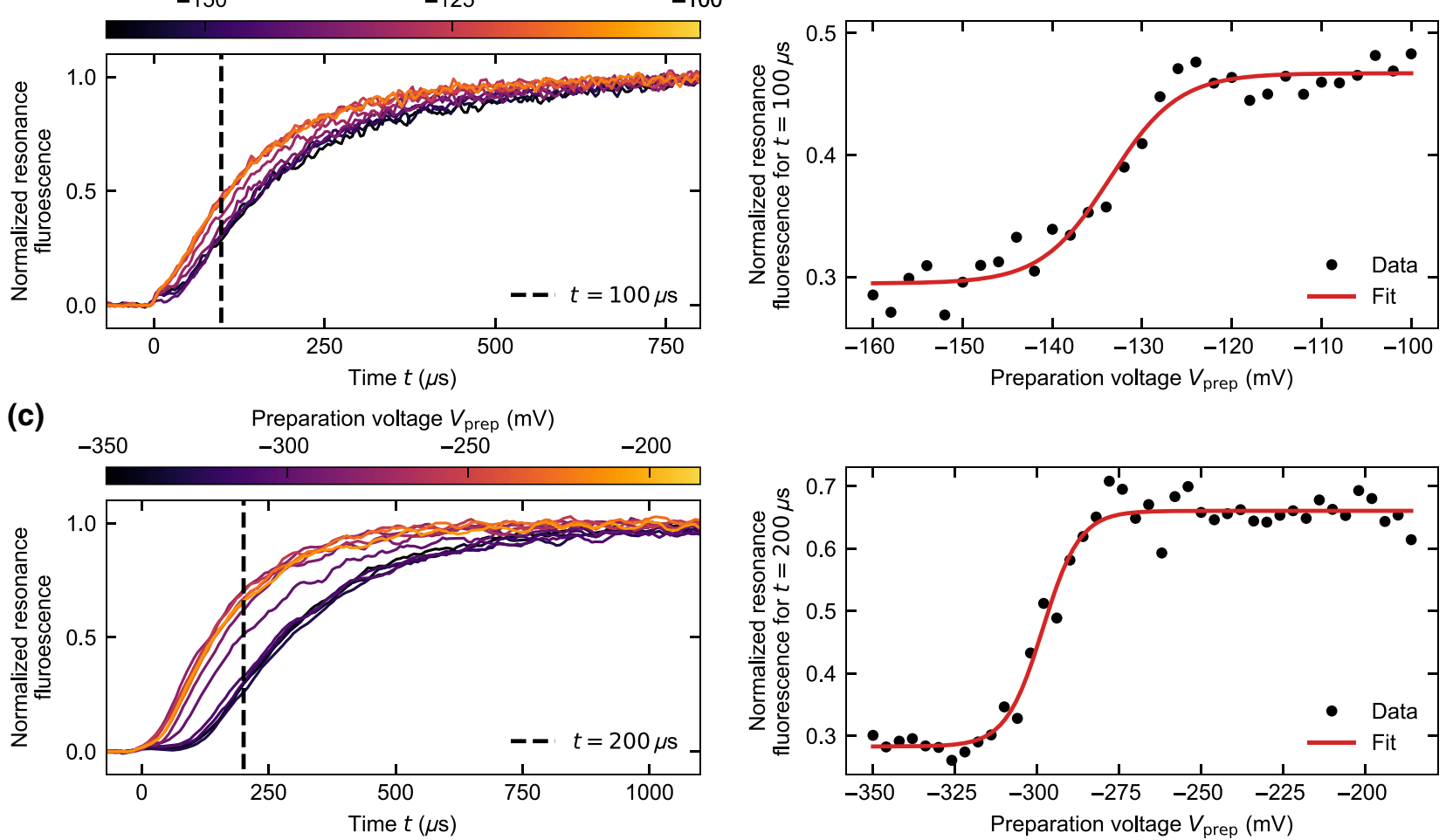

FIG. 10. Defect characterization by time-resolved resonance-fluorescence spectroscopy. Left panels show transients for selected preparation voltages. Dashed lines indicate time for amplitude evaluation. Right panels show corresponding amplitudes and fit. Data is shown for defects $A, C$, and $D$ in (a), (b), (c), respectively.

[1] S. T. Pantelides, The electronic structure of impurities and other point defects in semiconductors, Rev. Mod. Phys. 50, 797 (1978).

[2] B. I. Shklovskii and A. L. Efros, Electronic Properties of Doped Semiconductors (Springer, Berlin, Heidelberg, 1984).

[3] J. Houel, A. V. Kuhlmann, L. Greuter, F. Xue, M. Poggio, B. D. Gerardot, P. A. Dalgarno, A. Badolato, P. M. Petroff, A. Ludwig, D. Reuter, A. D. Wieck, and R. J. Warburton, Probing Single-Charge Fluctuations at a GaAs/AlAs Interface Using Laser Spectroscopy on a Nearby InGaAs Quantum dot, Phys. Rev. Lett. 108, 107401 (2012).
[4] A. V. Kuhlmann, J. Houel, A. Ludwig, L. Greuter, D. Reuter, A. D. Wieck, M. Poggio, and R. J. Warburton, Charge noise and spin noise in a semiconductor quantum device, Nat. Phys. 9, 570 (2013).

[5] A. N. Vamivakas, Y. Zhao, S. Falt, A. Badolato, J. M. Taylor, and M. Atature, Nanoscale Optical Electrometer, Phys. Rev. Lett. 107, 166802 (2011).

[6] C. T. Sah, L. Forbes, L. L. Rosier, and A. F. Tasch, Thermal and optical emission and capture rates and cross sections of electrons and holes at imperfection centers in semiconductors from photo and dark junction current and capacitance experiments, Solid-State Electron. 13, 759 (1970).

[7] D. V. Lang, Deep-level transient spectroscopy: A new method to characterize traps in semiconductors, J. Appl. Phys. 45, 3023 (1974). 
[8] E. F. Schubert, J. B. Stark, T. H. Chiu, and B. Tell, Diffusion of atomic silicon in gallium arsenide, Appl. Phys. Lett. 53, 293 (1988).

[9] C. Matthiesen, M. Geller, C. H. H. Schulte, C. Le Gall, J. Hansom, Z. Li, M. Hugues, E. Clarke, and M. Atatüre, Phase-locked indistinguishable photons with synthesized waveforms from a solid-state source, Nat. Commun. 4, 1600 (2013).

[10] A. V. Kuhlmann, J. H. Prechtel, J. Houel, A. Ludwig, D. Reuter, A. D. Wieck, and R. J. Warburton, Transformlimited single photons from a single quantum dot, Nat. Commun. 6, 8204 (2015).

[11] H. S. Nguyen, G. Sallen, C. Voisin, Ph. Roussignol, C. Diederichs, and G. Cassabois, Ultra-coherent single photon source, Appl. Phys. Lett. 99, 261904 (2011).

[12] K. D. Jöns, P. Atkinson, M. Müller, M. Heldmaier, S. M. Ulrich, O. G. Schmidt, and P. Michler, Triggered indistinguishable single photons with narrow line widths from site-controlled quantum dots, Nano Lett. 13, 126 (2012).

[13] N. Somaschi, V. Giesz, L. De Santis, J. C. Loredo, M. P. Almeida, G. Hornecker, S. L. Portalupi, T. Grange, C. Antón, J. Demory, C. Gómez, I. Sagnes, N. D. LanzillottiKimura, A. Lemaítre, A. Auffeves, A. G. White, L. Lanco, and P. Senellart, Near-optimal single-photon sources in the solid state, Nat. Photonics 10, 340 (2016).

[14] D. Huber, M. Reindl, Y. Huo, H. Huang, J. S. Wildmann, O. G. Schmidt, A. Rastelli, and R. Trotta, Highly indistinguishable and strongly entangled photons from symmetric GaAs quantum dots, Nat. Commun. 8, 15506 (2017).

[15] H. J. Kimble, The quantum internet, Nature 453, 1023 (2008).

[16] J. L. O’Brien, A. Furusawa, and J. Vučković, Photonic quantum technologies, Nat. Photonics 3, 687 (2009).

[17] C. H. Bennett and D. P. DiVincenzo, Quantum information and computation, Nature 404, 247 (2000).

[18] T. D. Ladd, F. Jelezko, R. Laflamme, Y. Nakamura, C. Monroe, and J. L. O'Brien, Quantum computers, Nature 464, 45 (2010).

[19] V. Scarani, H. Bechmann-Pasquinucci, N. J. Cerf, M. Dušek, N. Lutkenhaus, and M. Peev, The security of practical quantum key distribution, Rev. Mod. Phys. 81, 1301 (2009).

[20] Hoi-Kwong Lo, Marcos Curty, and Kiyoshi Tamaki, Secure quantum key distribution, Nat. Photonics 8, 595 (2014).

[21] C. L. Degen, F. Reinhard, and P. Cappellaro, Quantum sensing, Rev. Mod. Phys. 89, 035002 (2017).

[22] L. T. Hall, J. H. Cole, C. D. Hill, and L. C. L. Hollenberg, Sensing of Fluctuating Nanoscale Magnetic Fields Using Nitrogen-Vacancy Centers in Diamond, Phys. Rev. Lett. 103, 220802 (2009).

[23] J. R. Maze, P. L. Stanwix, J. S. Hodges, S. Hong, J. M. Taylor, P. Cappellaro, L. Jiang, M. V. Gurudev Dutt, E. Togan, A. S. Zibrov, A. Yacoby, R. L. Walsworth, and M. D. Lukin, Nanoscale magnetic sensing with an individual electronic spin in diamond, Nature 455, 644 (2008).

[24] J. M. Taylor, P. Cappellaro, L. Childress, L. Jiang, D. Budker, P. R. Hemmer, A. Yacoby, R. Walsworth, and M. D. Lukin, High-sensitivity diamond magnetometer with nanoscale resolution, Nat. Phys. 4, 810 (2008).
[25] P. Neumann, I. Jakobi, F. Dolde, C. Burk, R. Reuter, G. Waldherr, J. Honert, T. Wolf, A. Brunner, J. H. Shim, D. Suter, H. Sumiya, J. Isoya, and J. Wrachtrup, Highprecision nanoscale temperature sensing using single defects in diamond, Nano Lett. 13, 2738 (2013).

[26] L. P. McGuinness, Y. Yan, A. Stacey, D. A. Simpson, L. T. Hall, D. Maclaurin, S. Prawer, P. Mulvaney, J. Wrachtrup, F. Caruso, R. E. Scholten, and L. C. L. Hollenberg, Quantum measurement and orientation tracking of fluorescent nanodiamonds inside living cells, Nat. Nanotechnol. 6, 358 (2011).

[27] R. J. Schoelkopf, The radio-frequency single-electron transistor (RF-SET): A fast and ultrasensitive electrometer, Science 280, 1238 (1998).

[28] J. M. Elzerman, R. Hanson, L. H. Willems van Beveren, B. Witkamp, L. M. K. Vandersypen, and L. P. Kouwenhoven, Single-shot read-out of an individual electron spin in a quantum dot, Nature 430, 431 (2004).

[29] S. Gustavsson, R. Leturcq, B. Simovič, R. Schleser, T. Ihn, P. Studerus, K. Ensslin, D. C. Driscoll, and A. C. Gossard, Counting Statistics of Single Electron Transport in a Quantum dot, Phys. Rev. Lett. 96, 076605 (2006).

[30] A. N. Vamivakas, M. Atatüre, J. Dreiser, S. T. Yilmaz, A. Badolato, A. K. Swan, B. B. Goldberg, A. Imamoglu, and M. S. Ünlü, Strong extinction of a far-field laser beam by a single quantum dot, Nano Lett. 7, 2892 (2007).

[31] M. Hauck, F. Seilmeier, S. E. Beavan, A. Badolato, P. M. Petroff, and A. Hogele, Locating environmental charge impurities with confluent laser spectroscopy of multiple quantum dots, Phys. Rev. B 90, 235306 (2014).

[32] A. R. Peaker, V. P. Markevich, and J. Coutinho, Tutorial: Junction spectroscopy techniques and deep-level defects in semiconductors, J. Appl. Phys. 123, 161559 (2018).

[33] A. Chantre, G. Vincent, and D. Bois, Deep-level optical spectroscopy in GaAs, Phys. Rev. B 23, 5335 (1981).

[34] M. Geller, C. Kapteyn, L. Müller-Kirsch, R. Heitz, and D. Bimberg, $450 \mathrm{meV}$ hole localization in $\mathrm{GaSb} / \mathrm{GaAs}$ quantum dots, Appl. Phys. Lett. 82, 2706 (2003).

[35] M. Geller, A. Marent, E. Stock, D. Bimberg, V. I. Zubkov, I. S. Shulgunova, and A. V. Solomonov, Hole capture into self-organized InGaAs quantum dots, Appl. Phys. Lett. 89, 232105 (2006).

[36] P. W. Fry, I. E. Itskevich, D. J. Mowbray, M. S. Skolnick, J. J. Finley, J. A. Barker, E. P. O’Reilly, L. R. Wilson, I. A. Larkin, P. A. Maksym, M. Hopkinson, M. Al-Khafaji, J. P. R. David, A. G. Cullis, G. Hill, and J. C. Clark, Inverted Electron-Hole Alignment in InAs-GaAs Self-Assembled Quantum Dots, Phys. Rev. Lett. 84, 733 (2000).

[37] A. Kurzmann, B. Merkel, P. A. Labud, A. Ludwig, A. D. Wieck, A. Lorke, and M. Geller, Optical Blocking of Electron Tunneling Into a Single Self-Assembled Quantum dot, Phys. Rev. Lett. 117, 017401 (2016).

[38] H. Drexler, D. Leonard, W. Hansen, J. P. Kotthaus, and P. M. Petroff, Spectroscopy of Quantum Levels in ChargeTunable Ingaas Quantum Dots, Phys. Rev. Lett. 73, 2252 (1994).

[39] G. Snider, 1D Poisson/Schrödinger: A band diagram calculator, University of Notre Dame. 
[40] A. P. Mills Jr, L. N. Pfeiffer, K. W. West, and C. W. Magee, Mechanisms for si dopant migration in molecular beam epitaxy $\mathrm{Al}_{x} \mathrm{Ga}_{1-x} \mathrm{As}$, J. Appl. Phys. 88, 4056 (2000).

[41] Y. J. Chung, K. W. Baldwin, K. W. West, M. Shayegan, and L. N. Pfeiffer, Surface segregation and the al problem in GaAs quantum wells, Phys. Rev. Mater. 2, 034006 (2018).
[42] C. M. Wolfe, D. M. Korn, and G. E. Stillman, Silicon as a residual donor in high-purity GaAs, Appl. Phys. Lett. 24, 78 (1974).

[43] Khaled Karrai and Richard J. Warburton, Optical transmission and reflection spectroscopy of single quantum dots, Superlattices Microstruct. 33, 311 (2003). 